\title{
Possibilities of new data in hadroproduction ${ }^{1}$
}

\author{
Rajendran Raja \\ Fermilab, P.O. Box 500, Batavia, Illinois 60510, U.S.A
}

\begin{abstract}
We describe experiments that can produce data that is of relevance to hadronic shower simulation. In particular we describe the possibilities to dramatically improve the quality of the data available for improving hadronic shower simulators made possible with the upgraded Main Injector Particle production Experiment (MIPP). We describe the status of MIPP which has to date acquired 18 million events of particle interactions using $(5 \mathrm{GeV} / \mathrm{c}-120 \mathrm{GeV} / \mathrm{c}) \pi^{ \pm}, K^{ \pm}$and $p^{ \pm}$beams on various targets and the plans to upgrade the data acquisition speed of MIPP to make it run 100 times faster.
\end{abstract}

Keywords: Particle production, Calorimetry

PACS: $13.75 .-n ; 13.85 .-\mathrm{t}$

\section{INTRODUCTION}

We describe experiments that will produce data in the near future that is of relevance to hadronic shower simulators. The HARP experiment [1] has acquired data using pion and proton beams in the momentum range (3-15 GeV/c) over a wide variety of targets ranging from beryllium to lead. In addition they also had cryogenic targets of hydrogen, deuterium, oxygen and nitrogen. They have recently published their results on pion production on $\mathrm{p}-\mathrm{Al}$ collision [2] at $12.9 \mathrm{GeV} / \mathrm{c}$. More publications from the large amount of data collected (420 million events) by HARP will add significantly to our knowledge of hadroproduction in their momentum range. In addition, the NA49 experiment [3] has proposed an upgrade, which if approved, will provide data on proton and pion interactions on Carbon in the momentum range 30-158 GeV/c. In what follows, we describe the MIPP experiment [4], and the data taken so far and the proposal to upgrade the experiment to make it acquire data 100 times faster.

\section{CURRENT STATUS OF THE MIPP EXPERIMENT}

We give a status report on the MIPP experiment and its performance to date. MIPP is situated in the Meson Center beamline at Fermilab. It received approval [5] in November 2001 and has installed and operated both the experiment and a newly designed secondary beamline in the interim. It received its first beams in March 2004, had an engineering run to commission the detector in 2004 and had its physics data-taking run in the period January 2005-March 2006. The experiment is currently busy analyzing its data.

${ }^{1}$ Work supported by Fermi Research Alliance, LLC, under contract No. DE-AC02-07CH11359 with the U.S. Department of Energy. 
MIPP is designed primarily as an experiment to measure and study in detail the dynamics associated with non-perturbative strong interactions. It has nearly $100 \%$ acceptance for charged particles and excellent momentum resolution. Using particle identification techniques that encompass $d E / d x$, time-of-flight [6], Multi-Cell Čerenkov [7] and a Ring Imaging Čerenkov (RICH)detector [8], MIPP is designed to identify charged particles at the $3 \sigma$ or better level in nearly all of its final state phase space. MIPP has acquired data of high quality and statistics for beam momenta ranging from $5 \mathrm{GeV} / \mathrm{c}$ to $90 \mathrm{GeV} / \mathrm{c}$ for 6 beam species $\left(\pi^{ \pm}, K^{ \pm}\right.$and $\left.p^{ \pm}\right)$on a variety of targets as shown in Figure 1.

\begin{tabular}{|c|c|c|c|c|c|c|c|c|c|c|c|c|}
\hline \multirow{2}{*}{\multicolumn{3}{|c|}{$\begin{array}{c}\text { Data Summary } \\
27 \text { February } 2006 \\
\text { Target }\end{array}$}} & \multicolumn{10}{|c|}{$\begin{array}{c}\text { Acquired Data by Target and Beam Energy } \\
\text { Number of events, } \times 10^{6}\end{array}$} \\
\hline & & & \multicolumn{9}{|c|}{$\mathbf{E}$} & \multirow[b]{2}{*}{ Total } \\
\hline $\mathbf{Z}$ & Element & $\begin{array}{l}\text { Trigger } \\
\text { Mix }\end{array}$ & 5 & 20 & 35 & 40 & 55 & 60 & 65 & 85 & 120 & \\
\hline \multirow{3}{*}{$\mathbf{0}$} & Empty & Normal & & 0.10 & 0.14 & & & 0.52 & & & 0.25 & 1.01 \\
\hline & $K$ Mass & No Int. & & & & 5.48 & 0.50 & 7.39 & 0.96 & & & 14.33 \\
\hline & Empty LH & Normal & & 0.30 & & & & 0.61 & & 0.31 & & \multirow{2}{*}{7.08} \\
\hline $\mathbf{1}$ & LH & Normal & 0.21 & 1.94 & & & & 1.98 & & 1.73 & & \\
\hline \multirow{2}{*}{4} & \multirow{2}{*}{ Be } & $p$ only & & & & & & & & & 1.08 & \multirow{2}{*}{1.75} \\
\hline & & Normal & & & 0.10 & & & 0.56 & & & & \\
\hline \multirow{3}{*}{6} & $\mathrm{C}$ & Mixed & & & & & & 0.21 & & & & \multirow{2}{*}{1.33} \\
\hline & C $2 \%$ & Mixed & & 0.39 & & & & 0.26 & & & 0.47 & \\
\hline & NuMI & $p$ only & & & & & & & & & 1.78 & 1.78 \\
\hline 13 & Al & Normal & & & 0.10 & & & & & & & 0.10 \\
\hline \multirow{2}{*}{83} & \multirow{2}{*}{$\mathbf{B i}$} & $p$ only & & & & & & & & & 1.05 & \multirow{2}{*}{2.83} \\
\hline & & Normal & & & 0.52 & & & 1.26 & & & & \\
\hline 92 & $\mathbf{U}$ & Normal & & & & & & 1.18 & & & & 1.18 \\
\hline \multicolumn{3}{|c|}{ Total } & 0.21 & 2.73 & 0.86 & 5.48 & 0.50 & 13.97 & 0.96 & 2.04 & 4.63 & 31.38 \\
\hline
\end{tabular}

FIGURE 1. The data taken during the first MIPP run as a function of nucleus. The numbers are in millions of events. During the last month of the run, the Jolly Green Giant magnet coils developed shorts. This time was used to acquire data without the TPC for exploring the feasibility of measuring the charged kaon mass using the $\mathrm{RICH}$ radii.

An important aspect of MIPP data-taking was the measurement of particle production off the NuMI [9] target in order to minimize the systematics in the near/far detector ratio in the MINOS [9] experiment. MIPP also made measurements with proton beams off various nuclei for the needs of proton radiography [5].

Another physics motivation behind MIPP is to restart the study of non-perturbative QCD interactions. Currently available data are of poor quality, and sparsely populate the beam momentum, $p_{T}$, and atomic weight phase space that makes comparisons between different experiments difficult. The MIPP TPC [10] digitizes the charged tracks in three dimensions, obviating the need for track matching across stereo views. Coupled with the 
particle identification capability of MIPP, the data from MIPP would add significantly to our knowledge base of non-perturbative QCD. This would help test inclusive scaling relations and also scaling nuclear reactions.

\section{Experimental Setup}

We designed a secondary beam [11] specific to our needs. The resonantly extracted protons from the Fermilab Main Injector are transported down the Meson Center line. They impinge on a $20 \mathrm{~cm}$ long copper target producing secondary beam particles. This target is imaged onto an adjustable momentum selection collimator which controls the momentum spread of the beam. This collimator is re-imaged on to our interaction target placed next to the TPC. The beam is tracked using three beam chambers and identified using two differential Čerenkovs [12] filled with gas, the composition and the pressure of which can be varied within limits as needed depending on the beam momentum and charge.

Figure 2 shows the layout of the apparatus. The TPC sits in a wide aperture magnet (the Jolly Green Giant) which has a peak field of 0.7 tesla. Downstream of the TPC are a 96 mirror multi-cell Čerenkov detector filled with $C_{4} F_{10}$ gas, and a time of flight system. This is followed by a large aperture magnet (Rosie) which runs in opposite polarity (at -0.6 tesla) to the Jolly Green Giant to bend the particles back into the Ring Imaging Cerenkov counter. The RICH has $\mathrm{CO}_{2}$ as the radiator and an array of phototubes of 32 rows and 89 columns [13]. Downstream of the RICH we have an electromagnetic calorimeter [14] and a hadron calorimeter [15] to measure forward-going photons and neutrons. The electromagnetic calorimeter provides a means of distinguishing forward neutrons from photons and will also serve as a device to measure the electron content of our beam at lower energies, which will be useful for measuring cross sections.

MIPP uses $d E / d x$ in the TPC to separate pions, kaons and protons for momenta less than $\approx 1 \mathrm{GeV} / \mathrm{c}$ and the time of flight array of counters to do the particle identification for momenta less than $2 \mathrm{GeV} / \mathrm{c}$. The multi-cell Čerenkov detector [7] contributes to particle identification in the momentum range $\approx 2.5 \mathrm{GeV} / \mathrm{c}-14 \mathrm{GeV} / \mathrm{c}$ and the RICH [8] for momenta higher than this. By combining information from all counters, we get the expected particle identification separation for $K / p$ and $\pi / K$ as shown in Figure 3. It can be seen that excellent separation at the $3 \sigma$ or higher level exists for both $K / p$ and $\pi / p$ over almost all of phase space. Tracking of the beam particles and secondary beam particles is accomplished by a set of drift chambers [16] and proportional chambers [17] each of which have 4 stereo layers.

\section{Some results from Acquired data}

Figure 4 shows the pictures of reconstructed tracks in the TPC obtained during the data-taking run. The tracks are digitized and fitted as helices in three dimensions. Extrapolating three dimensional tracks to the other chambers makes the pattern recognition particularly easy. 


\section{MIPP \\ Main Injector Particle Production Experiment (FNAL-E907)}

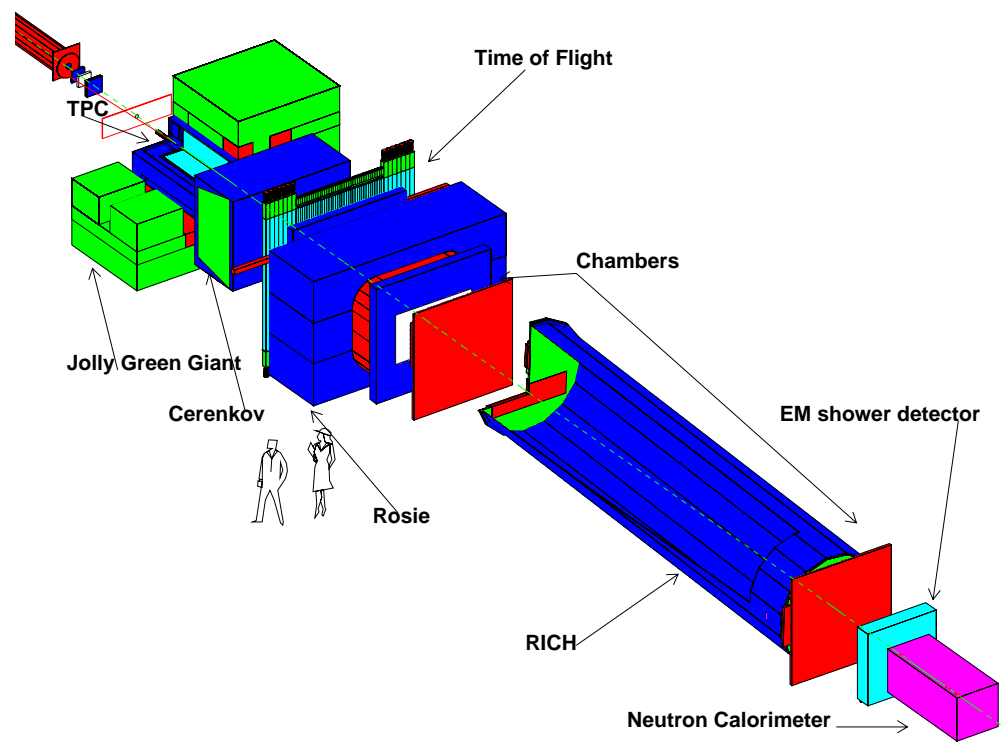

FIGURE 2. The experimental setup. The picture is a rendition in Geant3, which is used to simulate the detector.

Figure 5 shows the distribution of $d E / d x$ of tracks measured in the TPC as a function of the track momentum in a preliminary analysis of p-Carbon data. The TPC is capable of separating pions, protons and kaons in the momentum range below $\approx 1 \mathrm{GeV} / \mathrm{c}$. Figure 6 shows events with multiple rings in the RICH counter. Figure 7 shows the histogram of ring radii for a $+40 \mathrm{GeV}$ secondary beam. There is clean separation between pions, kaons and protons and their relative abundances [18] match expectations. Applying the particle identification trigger from the beam Čerenkovs enables us to separate the three particle species cleanly. The kaons which form $4 \%$ of the beam are cleanly picked out by the beam Čerenkov with very simple selection criteria. These can be made much more stringent with offline cuts to produce a very clean kaon beam.

The ring radius of the particle contains information on the mass of the particle. The pion and proton masses are very well known. The charged kaon mass, however, currently has measurement uncertainties of the order of $60 \mathrm{keV}$. Improving the precision of both charged kaon masses will pay dividends in rare $\mathrm{K}$ decay experiments involving charged kaons where the matrix elements depend on the kaon mass raised to large powers. Towards the end of our physics run, when the Jolly Green Giant magnet coils failed, we switched off the TPC and acquired data at the rate of $300 \mathrm{~Hz}$ to investigate how well 

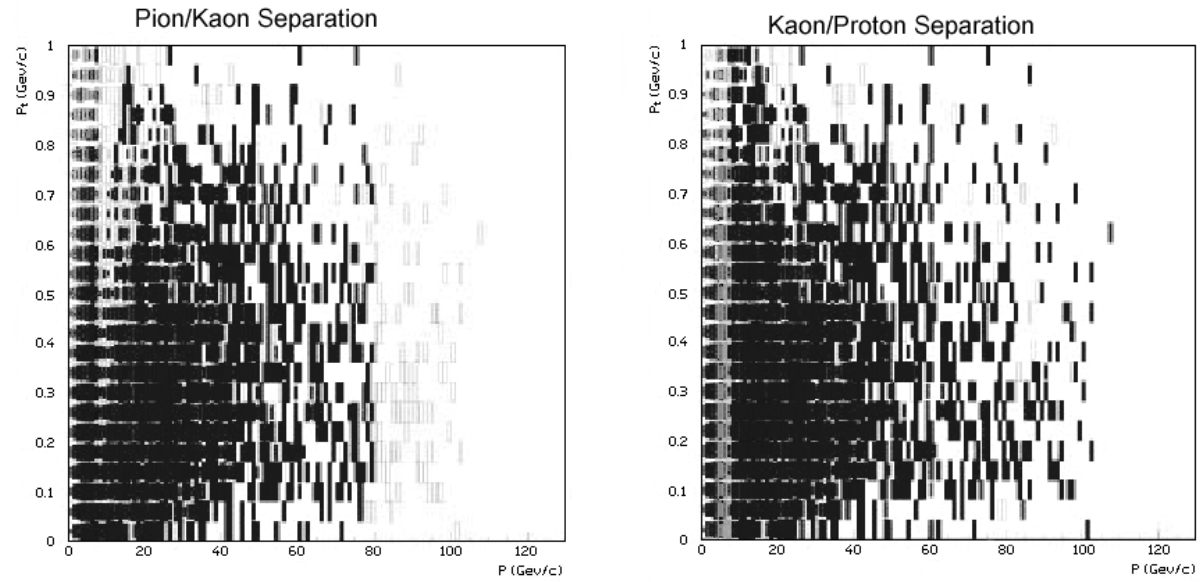

FIGURE 3. Particle identification plots for pion/kaon separation and for kaon/proton separation as a function of the longitudinal and transverse momentum of the outgoing final state particle. Black indicates separation at the $3 \sigma$ level or better and grey indicates separation at the $1-3 \sigma$ level. The boxes at largest values of the longitudinal momenta suffer from lack of kaon statistics.
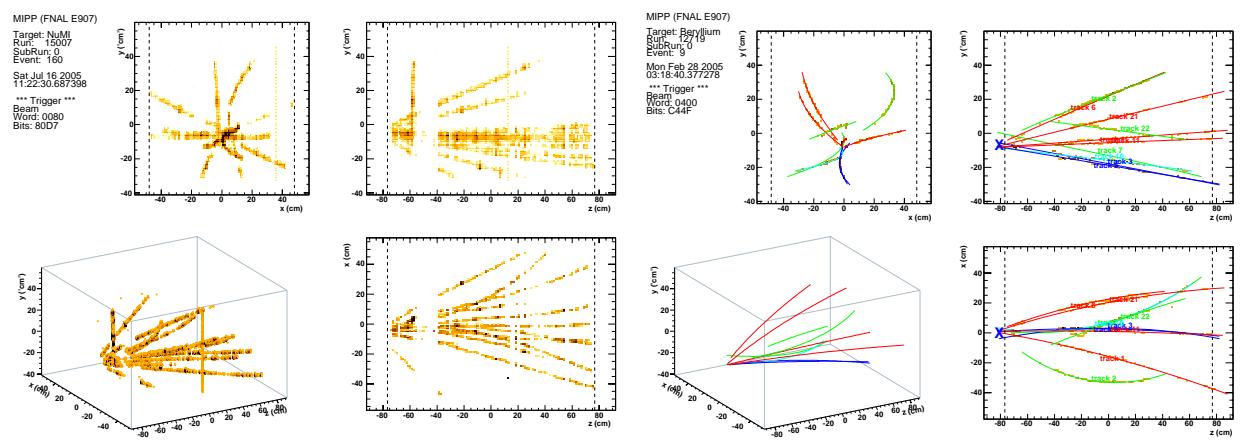

FIGURE 4. RAW and Reconstructed TPC tracks from two different events.
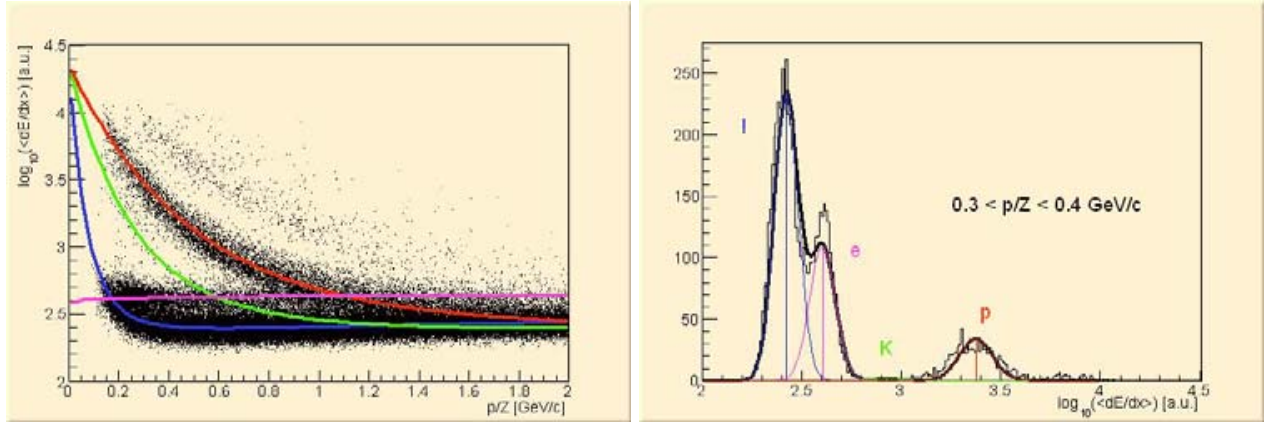

FIGURE 5. Preliminary $\mathrm{dE} / \mathrm{dx}$ distributions in the TPC The scatter plot shows the electron, pion, kaon and proton peaks in the distribution as a function of the lab momentum for $\mathrm{p}$-Carbon data. The second plot is the projection on the $\mathrm{dE} / \mathrm{dx}$ axis for a momentum slice $0.3 \mathrm{GeV} / \mathrm{c}$ to $0.4 \mathrm{GeV} / \mathrm{c}$.

we can measure the charged kaon mass. These events, whose statistics are indicated in 

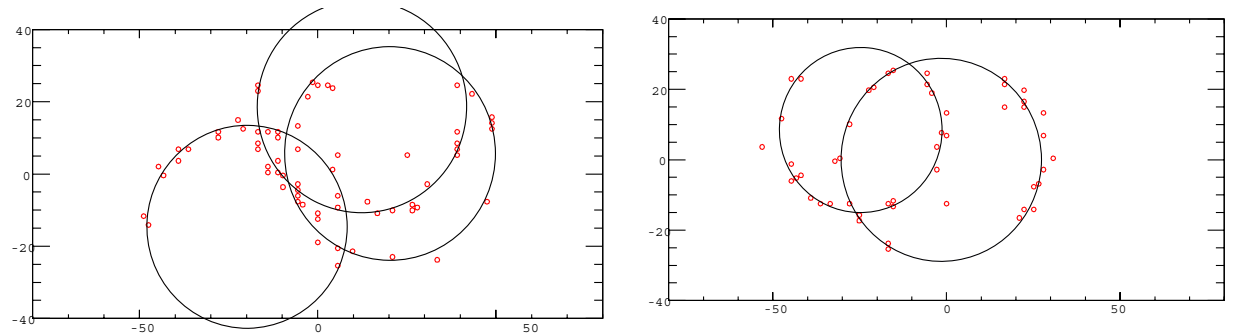

FIGURE 6. Examples of events with rings in the RICH counter for a $40 \mathrm{GeV} / \mathrm{c}$ beam. The $\mathrm{x}$ and $\mathrm{y}$ axes are in $\mathrm{cm}$.

Figure 1, are currently being analyzed to evaluate the systematics involved in such a measurement.
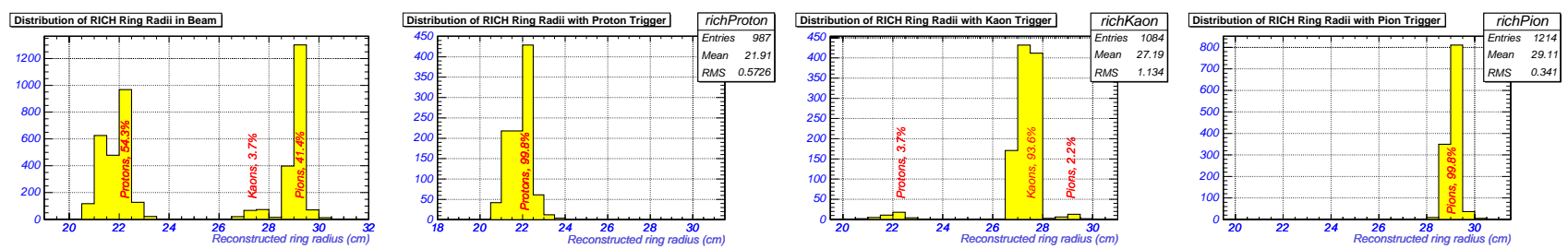

FIGURE 7. An example of a $40 \mathrm{GeV} / \mathrm{c}$ primary beam (non-interacting) trigger. The RICH identifies protons, kaons and pions by the ring radii. The beam Čerenkov detectors can be used to do the same. When the beam Čerenkov identification is used, one gets a very clean separation of pions, kaons and protons in the RICH.

\section{NuMI target measurements}

MIPP took 1.75 million events using $120 \mathrm{GeV} / \mathrm{c}$ primary beam protons impinging on the NuMI (spare) target. These events will play a crucial role in the prediction of neutrino fluxes in the NuMI beamline and will enable the MINOS experiment to control the systematics in the near/far detector ratios as well as helping them understand the near detector performance. Figure 8 shows a radiograph of the MIPP measurements of the MINOS target. The graphite slabs and cooling tubes can be seen. These events were obtained during the commissioning phase of this target measurement where the beam was not yet fully focused and aligned on the target. The 1.75 Million events on the NuMI target were obtained after the beam was aligned and centered on the target. Figure 9 shows the rich ring radii vs momentum of positive tracks originating from the NuMI target. Superimposed are the curves for known particles. This shows the excellent particle identification of the MIPP detector for forward going particles.

\section{MIPP UPGRADE PROPOSAL}

In October 2006, MIPP proposed to upgrade its detector by increasing the data acquisition speed of its TPC from $20 \mathrm{~Hz}$ to $3000 \mathrm{~Hz}$ using the ALTRO/PASA chips developed 


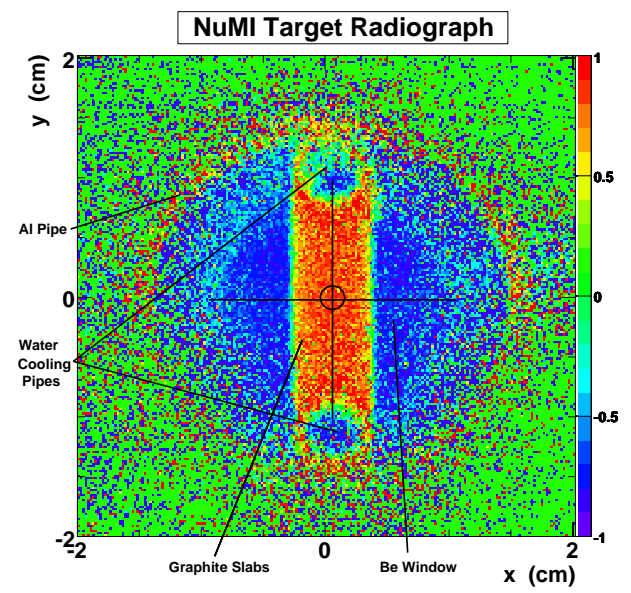

FIGURE 8. Radiograph of the MINOS target. The beam direction is perpendicular to the paper.

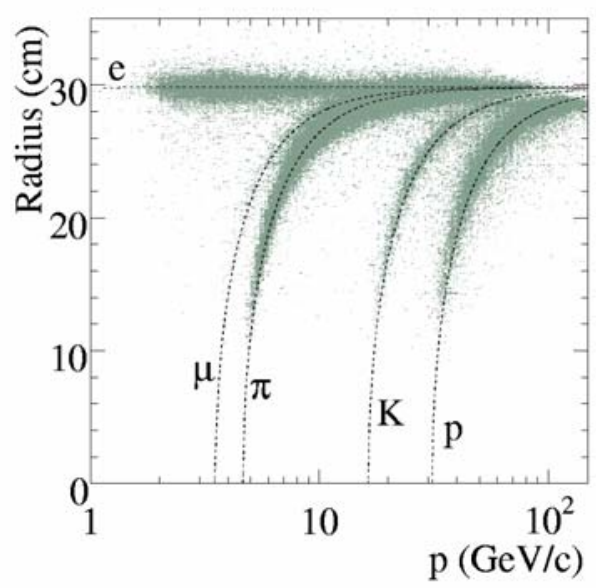

FIGURE 9. Preliminary data of RICH ring radii of positive tracks from the NuMI target vs momentum. Superimposed are the expected curves for $e, \mu, K$ and $p$ particles.

for the ALICE collaboration [19]. With this large factor in data acquisition speed, it becomes possible to acquire 5 million events in a single day of running. The electronics of the rest of the detector will also be upgraded to run at this speed. We propose to use a hemisphere of the plastic ball detector [20] to measure the target recoil particles. This enables us to measure particle production on 30 of the most common nuclei found in particle physics detectors and improve the hadronic shower simulator programs. It will also permit us to study non-perturbative QCD in unprecedented detail. The baryon resonance spectrum can be investigated up to $3 \mathrm{GeV} / \mathrm{c}^{2}$, using pion and kaon beams in the momentum range $1 \mathrm{GeV} / \mathrm{c}-5 \mathrm{GeV} / \mathrm{c}$. The decision on the upgrade proposal has been deferred till analysis results from the present run are available and the collaboration has been strengthened further. 


\section{Upgrade Run Plan}

We propose to conduct the upgrade running in three phases. In the first phase, we will acquire data on the neutrino targets, liquid nitrogen and take particle production measurements on 12 thin nuclear targets (5 million events per nucleus) which will improve the quality of hadronic shower simulation programs. The number of events acquired in each target is indicated in table 1.

TABLE 1. Phase 1 Run Plan.

\begin{tabular}{ccc}
\hline Target & $\begin{array}{c}\text { Events } \\
\text { (Millions) }\end{array}$ & $\begin{array}{c}\text { Running Time } \\
\text { (Days) }\end{array}$ \\
\hline NuMI target 1 & 10 & 2 \\
NuMI target 2 & 10 & 2 \\
Liquid Hydrogen & 20 & 4 \\
Liquid Nitrogen & 10 & 2 \\
12 Nuclei & & \\
$D_{2}, \mathrm{Be}, \mathrm{C}, \mathrm{Al}, \mathrm{Si}, \mathrm{Hg}$, & & \\
Fe,Ni,Cu,Zn,W,Pb & 60 & 12 \\
Total Events & 110 & 22 \\
\hline
\end{tabular}

TABLE 2. Phase 2 Run Plan.

\begin{tabular}{ccc}
\hline Target & $\begin{array}{c}\text { Events } \\
\text { (Millions) }\end{array}$ & $\begin{array}{c}\text { Running Time } \\
\text { (Days) }\end{array}$ \\
\hline 18 Nuclei & & \\
Li,B, $\mathrm{O}_{2}, \mathrm{Mg}, \mathrm{P}$, & & \\
S,Ar,K,Ca,Ni,Nb,Ag, & & 18 \\
Sn,Pt,Au,Pb,Bi,U & 90 & \\
10 Nuclei B-list & & \\
Na,Ti, V, Cr,Mn,Mo, & & 10 \\
I, Cd, Cs, Ba & 50 & 28 \\
Total Events & 140 & \\
\hline
\end{tabular}

During phase 2, we plan to complete the remaining 18 nuclei of the A-List, as detailed in the section on hadronic shower simulation and then proceed with the B-list if there is need. The second phase of running is detailed in table 2.

During phase 3, we plan to go into the tagged neutral beam mode, where we run the liquid hydrogen target and allow the ILC calorimetry to run simultaneously in place of the MIPP calorimeter to study the response of the ILC calorimeters to tagged neutral beams.

\section{HADRONIC INTERACTION LIBRARY}

With the upgrade, MIPP can change the landscape of data available for hadronic shower simulations by providing it in the form of a random access database which stores the four vectors and identities of the final state particles. With 6 million events per nucleus, distributed in ten beam momentum bins and six beam species and 100,000 events per bin, it is possible store this information in approximately 36 Gbytes of disk space. The data can be acquired in 36 calendar days of running. Such a random access library can 
be used directly by shower simulation programs or it can also be used to parametrize the models used in the programs currently. The former approach requires the use of a neutral particle algorithm which can be implemented assuming that the neutral particle rates are the average of the two charged particle states $\left(\right.$ e.g $\pi^{0}=\left(\pi^{+}+\pi^{-}\right) / 2$ and $K^{0}=\left(K^{+}+K^{-}\right) / 2$. Individual events can be picked in the appropriate reaction being simulated in the shower at a center of mass energy close to the one in the simulation and the center of mass longitudinal momenta of the events can then be scaled to match the event being simulated. It is possible to arrive at parametrisations of multiplicities as a function of $\sqrt{(s)}$ and this feature can be used to extrapolate the data to simulate showers at energies higher than those available in the data.

We plan to index each event by its missing mass (mass carried away by neutral particles). This will enable the simulation of neutral particles since we can look up events with small missing mass at the appropriate center of mass energy and turn the charged particles into neutral particles to simulate the neutral deposition.

Such a database of events can also act as a source of data for people formulating theoretical models of non-perturbative QCD interactions in the future.

\section{CONCLUSIONS}

MIPP has acquired high quality particle production data which it is busy analyzing. The MIPP upgrade will improve the quality and statistics of data available for hadronic shower programs by an order of magnitude.

\section{REFERENCES}

1. Information on the Harp experiment may be found at http://harp.web.cern.ch/harp/

and also in review talks such as "HARP: A Hadron Production Experiment", E.Radicioni for the collaboration, hep-ex/0206028.

2. "Measurement of the production cross-section of positive pions in p-Al collisions at 12.9-GeV/c", HARP collaboration, Nucl. Phys. B732:1-45,2006.

3. "A New SPS programme", NA49 future collaboration, M.Gazdzicki for the collaboration, nuclex/0612007.

4. The main MIPP web page is at http://ppd.fnal.gov/experiments/e907/. Information on the MIPP collaboration may be found using links there.

5. The MIPP proposal and Addendum to the proposal may be found at http://ppd.fnal.gov/experiments/e907 /Proposal/E907_Proposal.html

6. The Time of Flight detector was fabricated by MIPP and consists of an array of $10 \mathrm{~cm} \times 10 \mathrm{~cm}$ scintillators and $5 \mathrm{~cm} \times 5 \mathrm{~cm}$ scintillators. See http://ppd.fnal.gov/experiments/e907

/TOF/TOF.html for a detailed description of the detector.

7. The multi-cell Čerenkov detector was first used in BNL experiment E766, then in Fermilab experiment E690 followed by other BNL experiments.

8. The details of the SELEX RICH construction and performance may be found at J. Engelfried et al., Nucl. Instr. and Meth. A43,53(1999). We have replaced the front end electronics, and done extensive work on the safety systems. MIPP uses $\mathrm{CO}_{2}$ gas as the radiator for the RICH.

9. NuMI stands for Neutrinos at the Main Injector and refers to the Fermilab Main Injector neutrino beam. MINOS is the first experiment to utilize this beam MINOS proposal may be found at "P-875: A 
long baseline neutrino oscillation experiment at Fermilab", E. Ables et al.; FERMILAB-PROPOSALP875,(1995). See also their website at http://www-numi.fnal.gov/

10. The TPC was built by the BEVALAC group at Berkeley in the 1990's and used effectively at several Brookhaven experiments (e.g. E910) and then donated to Fermilab by LBNL for use in MIPP. See, G. Rai et al., IEEE Trans.Nucl.Sci.37,56(1990); LBL-28141.

11. "Beamline design for particle production Experiment, E907 at FNAL", C. Johnstone et al., Proceedings of the PAC03 conference. So successful has the performance of this beamline been that the design has been adopted for use in the M-Test beamline upgrade for providing testbeams at Fermilab.

12. For more details on the beam Čerenkov system, see http://ppd.fnal.gov/experiments/e907/Beam

/BeamCerenkov/BeamCerenkov.html

13. In 2004, we lost $20 \%$ of the phototubes in the RICH due to a fire in one of the phototube bases. This does not impact adversely on our pattern recognition, since the Čerenkov angle is large and there is plenty of light over most of our momentum range.

14. The EM calorimeter was fabricated by MIPP and uses lead as the radiator and an array of proportional tubes with $2.54 \mathrm{~cm}$ wire spacing as the readout. It has 10 radiation lengths and has 10 longitudinal segments.

15. Theiron/scintillator fiber hadron calorimeter is recycled from the HyperCP collaboration. It has 9.7 interaction lengths and has 4 longitudinal and 2 transverse segmentations.

16. We have reused beam and drift chambers from the E690 collaboration. D.C.Christian et al., Nucl. Instr. and Meth. A345,62 (1994).

17. The large proportional chambers straddling the RICH find their use again after having been used by numerous previous experiments. M. De Palma et al., Nucl. Instr. and Meth. 216 (1983) 393-397.

18. A. Malensek, Fermilab Technical Memo FN-341, 1981. (unpublished).

19. The details of the MIPP upgrade Proposal (P-960) can be found at http://ppd.fnal.gov/experiments/e907/notes/

MIPPnotes/public/pdf/MIPP0138/MIPP0138.pdf

20. The plastic ball detector was constructed by GSI Darmstadt physicists in the early 1980's. See R. Albrecht et. al., CERN Report CERN/SPSC/85-39 (august 1985), GSI Report GSI-85-32 (August 1985), for more details. 